QIANG TU, Ph.D. Candidate ${ }^{1}$

E-mail: dqtu_1991@163.com

LIN CHENG, Ph.D. ${ }^{1}$

(Corresponding author)

E-mail: gist@seu.edu.cn

DAWEI LI, Ph.D.1,2,3

E-mail: lidawei@seu.edu.cn

JIE MA, Ph.D. Candidate ${ }^{1}$

E-mail: majie9001@163.com

CHAO SUN, Ph.D. ${ }^{4}$

E-mail: sunchao900217@126.com

${ }^{1}$ School of Transportation, Southeast University

Si Pai Lou \#2, Nanjing 210096, China

2 Jiangsu Key Laboratory of Urban ITS, Southeast University

Si Pai Lou \#2, Nanjing 210096, China

${ }^{3}$ Collaborative Innovation Center of Modern Urban Traffic,

Southeast University

Si Pai Lou \#2, Nanjing 210096, China

${ }^{4}$ School of Automotive and Traffic Engineering

Jiangsu University

Xuefu Road \#301, Zhengjiang 212013, China
Science in Traffic and Transport

Original Scientific Paper

Submitted: 30 Oct. 2017

Accepted: 21 Sep. 2018

\title{
TRAFFIC PARADOX UNDER DIFFERENT EQUILIBRIUM CONDITIONS CONSIDERING ELASTIC DEMAND
}

\begin{abstract}
Traffic paradox is an important phenomenon which needs attention in transportation network design and traffic management. Previous studies on traffic paradox always examined user equilibrium (UE) or stochastic user equilibrium (SUE) conditions with a fixed traffic demand (FD) and set the travel costs of links as constants under the SUE condition. However, traffic demand is elastic, especially when there are new links added to the network that may induce new traffic demand, and the travel costs of links actually depend on the traffic flows on them. This paper comprehensively investigates the traffic paradox under different equilibrium conditions including the user equilibrium and the stochastic user equilibrium with a fixed and elastic traffic demand. Origin-destination (OD) mean unit travel cost (MUTC) has been chosen as the main index to characterize whether the traffic paradox occurs. The impacts of travelers' perception errors and travel cost sensitivity on the occurrence of the traffic paradox are also analyzed. The conclusions show that the occurrence of the traffic paradox depends on the traffic demand and equilibrium conditions; higher perception errors of travelers may lead to a better network performance, and a higher travel cost sensitivity will create a reversed traffic paradox. Finally, several appropriate traffic management measures are proposed to avoid the traffic paradox and improve the network performance.
\end{abstract}

\section{KEY WORDS}

traffic paradox; user equilibrium; stochastic user equilibrium; fixed demand; elastic demand; method of successive average;

\section{INTRODUCTION}

Traffic paradox has been widely examined since it was first proposed by Braess in 1968 [1]. As it is defined under the user equilibrium with the fixed demand (UE-FD) condition, the addition of a new link may result in a higher total network travel cost. Braess' paradox occurs because travelers attempt to minimize their own travel time while ignoring the effect of their decisions on other travelers, which is decided by the user equilibrium condition. Following this idea, many studies about Braess' paradox have been conducted [2-7]. For example, Pas and Principio found that the occurrence of the paradox depends on the traffic demand, as it only occurs in a certain demand range [8], Yang and Bell proposed a traffic paradox based on network capacity [9], and Korilis made some suggestions on how to avoid Braess' paradox [10]. However, they are all conducted under the UE condition. Braess' paradox under the SUE can refer to Zhao et al. [11-12], but they set the travel costs of links as constants rather than as flow-dependent values. Besides, some researchers extended Braess' paradox to dynamic traffic assignment problems [13-15].

According to reviews of previous studies, we know that different equilibrium conditions (i.e., traffic assignment models) will impact whether the traffic paradox occurs and how it works (e.g. traffic paradox does not occur under the system optimization condition, for its objective function is a minimum total travel cost). 
Depending on whether the travelers' perceived randomness is observed, the equilibrium conditions can be classified as UE and SUE. On the other hand, depending on whether the observed traffic demand is elastic or not, the equilibrium conditions can be classified as fixed demand (FD) and elastic demand (ED). All four conditions (i.e., UE-FD, SUE-FD, UE-ED and SUEED) will be examined in this paper.

The common index which characterizes whether Braess' paradox occurs is the difference of total travel cost (TTC) between the original network and the new network with additional links [12, 15, 16]. However, it may not apply to the case of the ED condition, because the addition of links may induce higher traffic demand and make the TTC higher. In this paper, in order to achieve an average distribution of the TTC, we have chosen the mean unit travel cost (MUTC) as the main index. However, the indexes of the TTC and the traffic demand (only under elastic demand condition) are still analyzed.

It is clear that the traffic demand will determine whether the traffic paradox occurs when the link travel cost function and equilibrium conditions are confirmed [8]. And the traffic paradox occurs always in a congested network, namely the condition when the traffic demand is high. Furthermore, we will investigate the range of the traffic demand where the traffic paradox occurs under different equilibrium conditions and give some new insights. The impact of the parameters (i.e., travelers' perception errors and travel cost sensitivity) on the occurrence of the traffic paradox will also be examined.

The basic parameters of the network to be studied in this paper are introduced in the next section. After that, the traffic paradox with the FD and the ED are analyzed in Sections 3 and 4, respectively. Finally, conclusions about the main results of this paper are presented.

\section{BASIC DESCRIPTION}

The networks shown in Figure 1 are considered to be the example networks, which are essentially the same as the one used by Braess and widely used in other studies about the traffic paradox.

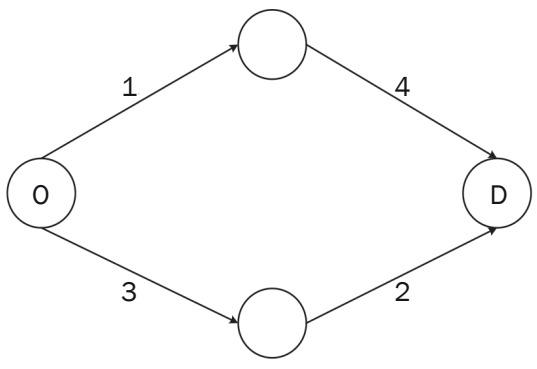

a) Four-link network
Figure 1a depicts a simple network including only one OD pair connected by two paths and four links. Path 1 consists of links 1 and 4; path 2 consists of links 3 and 2 .

In order to improve the network performance and reduce delay, link 5 is added to the original network to construct a new five-link network shown in Figure 1 b. Links 3, 5 and 4 form the new path 3 .

As in the classical Braess' example, the travel cost functions for 5 links are assumed to be linear as follows:

$$
\begin{aligned}
& t_{1}=50+x_{1} \\
& t_{2}=50+x_{2} \\
& t_{3}=10 x_{3} \\
& t_{4}=10 x_{4} \\
& t_{5}=10+x_{5}
\end{aligned}
$$

where $t_{a}$ is the travel cost on link $a ; x_{a}$ is the flow on link $a$;

Based on the relationship between links and paths, the travel cost of three paths in networks are:

$$
\begin{aligned}
& c_{1}=t_{1}+t_{4} \\
& c_{2}=t_{2}+t_{3} \\
& c_{3}=t_{3}+t_{4}+t_{5}
\end{aligned}
$$

Note that the flows and travel cost on paths 1 and 2 are equal to the symmetrical property of both networks. Additionally, the flows on paths and links are necessary to satisfy the following flow conservation conditions.

In a four-link network, they are:

$x_{1}^{4}=x_{2}^{4}=x_{3}^{4}=x_{4}^{4}=f_{1}^{4}=f_{2}^{4}$

In a five-link network, they are:

$$
\begin{aligned}
& x_{1}^{5}=f_{1}^{5} \\
& x_{2}^{5}=f_{2}^{5} \\
& x_{3}^{5}=f_{2}^{5}+f_{3}^{5} \\
& x_{4}^{5}=f_{1}^{5}+f_{3}^{5} \\
& x_{5}^{5}=f_{3}^{5}
\end{aligned}
$$

The traffic demand of the OD is set as q. Assuming the capacity of every link is equal to 10 , it can be deduced that the capacity of two networks is 20 and

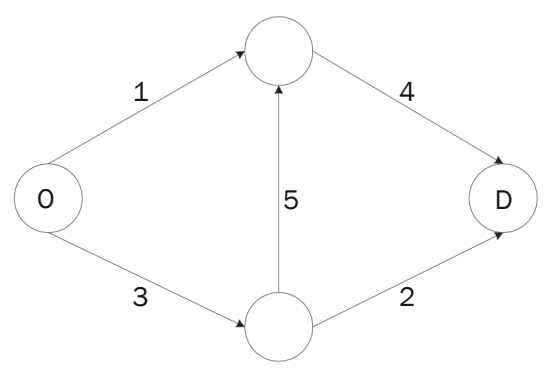

b) Five-link network

Figure 1 - Example networks 
$\left(20-x_{5}^{5}\right)$ in four-link and five-link networks, respectively [9]. Since link flow is not larger than its capacity $\left(0 \leq x_{5}^{5} \leq 10\right)$, the capacity of a five-link network falls within the interval of $[10,20]$. According to capacity constraints, the maximum values of $q$ (set as $q_{\max }$ ) are not larger than the capacity of networks. However, if traffic demand $q$ exceeds 10 , and reaches 11 , for example, one possible condition is that 10 travelers choose path 3 , and the remaining 1 traveler cannot be routed to the five-link network. To avoid this condition, we set $q_{\max }=10$ to guarantee that the traffic demand can never exceed the capacity of networks.

Apart from that, the traffic demand should satisfy the flow conservation condition as follows:

$q=\sum_{k} f_{k}, \quad \forall k \in K$

where $K$ represents a set of paths.

In previous studies, under the fixed demand condition, if the addition of link 5 results in the increase of the TTC of a network, the traffic paradox occurs. However, under the elastic demand condition, the addition of links may induce a higher traffic demand to make the TTC higher, which makes the index of the TTC not applicable under this condition, and the index of travel cost per traveler defined as the MUTC more meaningful. Let $T^{4}$ and $T^{5}$ represent the MUTC in a four-link and five-link network, and their expressions are:

$$
\begin{aligned}
& T^{4}=\frac{\sum_{a=1}^{4} x_{a}^{4} \cdot t_{a}^{4}}{q^{4}}=\frac{\sum_{k=1}^{2} f_{k}^{4} \cdot c_{k}^{4}}{q^{4}} \\
& T^{5}=\frac{\sum_{a=1}^{5} x_{a}^{5} \cdot t_{a}^{5}}{q^{5}}=\frac{\sum_{k=1}^{2} f_{k}^{5} \cdot c_{k}^{5}}{q^{5}}
\end{aligned}
$$

If $T^{5}>T^{4}$, the traffic paradox occurs. For convenience, we set $\Delta T=T^{5}-T^{4}$. Accordingly, $\Delta T>0$ represents the occurrence of the traffic paradox. Due to $q^{5}=q^{4}$ under the FD condition, the results of solving $q^{5} T^{5}>q^{4} T^{4}$ and $T^{5}>T^{4}$ are the same, so the indexes of TTC and MUTC are equivalent. We have chosen the MUTC as the main index to characterize whether the traffic paradox occurs, because it is applicable for both the FD and the ED conditions.

\section{FIXED DEMAND}

\subsection{User equilibrium (UE)}

The UE is a network state of each OD pair in which no traveler can reduce his or her travel cost by unilaterally changing paths. In other words, all used OD paths have an equal and minimum travel cost, and no unused route has a lower travel cost, i.e.:

$$
\begin{aligned}
& c_{k}=u, \text { if } f_{k}>0 ; \forall k \in K \\
& c_{k} \geq u, \text { if } f_{k}=0 ; \forall k \in K
\end{aligned}
$$

where $c_{k}$ is the travel cost on path $k$, and $u$ denotes the minimum OD path travel cost.

In a four-link network, by taking the advantage of symmetry, we can easily solve the equilibrium condition (Equation 7) to obtain the expression of $T^{4}$.

$T^{4}=\frac{11 q}{2}+50$

In a five-link network, the usage of paths will vary with the range of the traffic demand, which makes it more complex to obtain the expression of $T^{5}$, so we use the following process:

Step 1, assuming all paths are used, according to Equation 7, we have:

$c_{1}=c_{2}=c_{3}$

Solving Equations 1-5 and 9, we can obtain the expression of $f_{k}$ :

$f_{1}=\frac{11 q-40}{13}$

$f_{2}=\frac{11 q-40}{13}$

$f_{2}=\frac{80-9 q}{13}$

Step 2, if a path is unused, the flow on it is equal to zero. By solving $f_{k} \leq 0$, we can figure out which path is used and the corresponding range of the traffic demand in Table 1.

Step 3, according to Equation 7 and Table 1, by solving Equations 1-6, we can derive the expression of $T^{5}$ as follows:

Table 1 - The usage of paths in different ranges of traffic demand

\begin{tabular}{|c|c|c|c||}
\hline \multirow{2}{*}{ Path } & \multicolumn{3}{|c|}{ Traffic demand range } \\
\cline { 2 - 4 } & $q \leq \frac{40}{11}$ & $\frac{40}{11}<q<\frac{80}{9}$ & $q \geq \frac{80}{9}$ \\
\hline \hline 1 & $\times$ & $\sqrt{ }$ & $\sqrt{ }$ \\
\hline 2 & $\times$ & $\sqrt{ }$ & $\sqrt{ }$ \\
\hline 3 & $\sqrt{ }$ & $\sqrt{ }$ & $\times$ \\
\hline
\end{tabular}

$\times$ represents unused, $\sqrt{ }$ represents used

$T^{5}=\left\{\begin{array}{l}21 q+10 . \text { if } q \leq \frac{40}{11} \\ \frac{31 q+1010}{13} \text {.if } \frac{40}{11}<q<\frac{80}{9} \\ \frac{11 q}{2}+50 . \text { if } q \geq \frac{80}{9}\end{array}\right.$

According to Equations 8 and 11, the relationship between $\Delta T$ and the traffic demand under the UE-FD condition is shown in Figure 2, and the range of the traffic demand (RTD) where the traffic paradox occurs can be accurately determined as $[2.58,8.89]$. In other words, when the traffic demand is lower than 2.58 or higher than 8.89, the traffic paradox will not occur. 


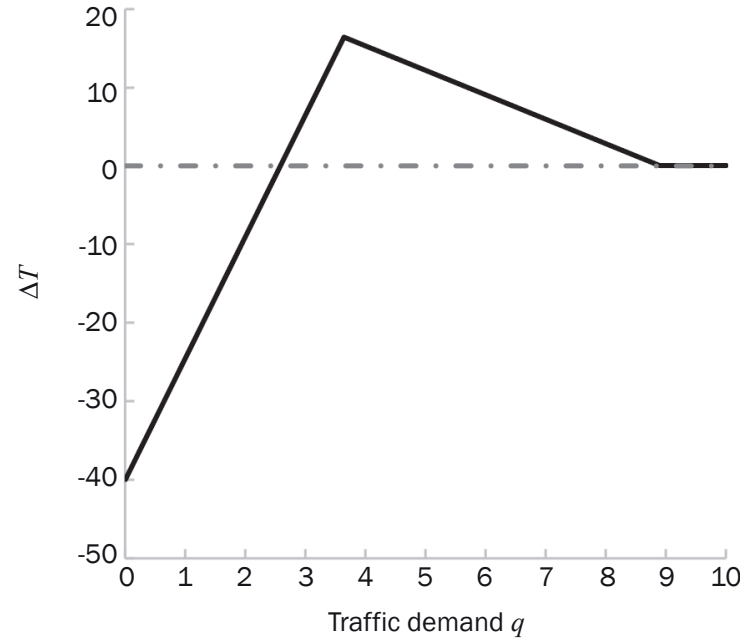

Figure 2 - The values of $\triangle T$ for variable $q$ under UE-FD

\subsection{Stochastic user equilibrium (SUE)}

Actually, travelers cannot know the travel cost accurately. They choose paths depending on their perceived travel cost on paths. The perceived travel cost is assumed to be:

$$
\tilde{c}_{k}=c_{k}+\xi_{k}
$$

where $\tilde{c}_{k}$ represents the perceived travel cost on path $k$, which is a random variable; $c_{k}$ is the actual OD travel cost on path $k ; \xi_{k}$ is a random error term associated with the path observed and its expectation is assumed to be 0 [17]. This means that the path will be chosen if its travel cost is perceived to be the lowest among all the alternative paths. The probability of choosing such a path can be expressed as follows:

$$
P_{k}=\operatorname{Pr}\left(c_{k}<c_{l}, \forall l \in K\right)
$$

where $P_{k}$ is the probability of choosing path $k$.

Different distributions of the examined travel time result in different models of the stochastic network loading. The Logit model has widely been used in discrete choice. In this paper, the same Logit model is utilized, in which utility terms are supposed to be independently and identically Gumbel-distributed. The choice probability is then obtained through the following:

$P_{k}=\frac{\exp \left(-\theta c_{k}\right)}{\sum_{l \in K} \exp \left(-\theta c_{l}\right)}$

where $\theta$ is a positive dispersion parameter, which can be interpreted as the degree of travelers' perception errors. A higher value of $\theta$ indicates that travelers have a more accurate perception of the travel cost or a higher-quality information on the traffic network condition [18], i.e., when $\theta \rightarrow+\infty$, the SUE is equal to the UE. On the contrary, a lower one indicates that the perceived error is greater, i.e., when $\theta \rightarrow 0$, the travelers believe that there is no difference in the travel cost of paths on a network, and that flows on every path are equal.

Hence, the probability of selecting each alternative path is specified, and the path flow assigned accordingly can be calculated. The path flow is obtained though the following:

$f_{k}=q \cdot P_{k} ; \quad \forall k \in K$

In a four-link network, though the observed travel cost is stochastic, due to the symmetrical property of the network, the flows and costs on path 1 and 2 are still equal, and the expression of $T^{4}$ is the same as that under the UE-FD which is $T^{4}=\frac{11 q}{2}+50$.

In a five-link network, since the analytical expression of $T^{5}$ cannot be definitely written, a route-based method of successive average (MSA) is developed to solve the SUE condition and obtain $T^{5}$.

Step 1. (Initialization) Set tolerance error $\varepsilon$, set the working path $K$, initial traffic flow $f_{k}^{(0)}$ and $x_{a}^{(0)}$ based on a free flow travel time, and initial iteration number $n=1$,

Step 2. (Update travel cost) Use Equations 1-4 to update $c_{k}^{(n)}$ and $t_{a}^{(n)}$,

Step 3. (Update traffic flow) Use Equations 14-15 to calculate the auxiliary path flow $d_{k}^{(n)}$ to find the iteration direction $\left(d_{k}^{(n)}-f_{k}^{(n)}\right)$, then update the traffic flow $f_{k}^{(n+1)}=f_{k}^{(n)}+\frac{1}{n}\left(d_{k}^{(n)}-f_{k}^{(n)}\right)$,

Step 4. (Check convergence) If $\frac{\sqrt{\sum_{k \in K}\left(f_{k}^{(n+1)}-f_{k}^{(n)}\right)^{2}}}{\sum_{k \in K} f_{k}^{(n)}} \leq \varepsilon$, then stop, $f_{k}^{(n+1)}$ is the optimal solution. Otherwise, set $n:=n+1$, and go to step 2 ,

Step 5. (Calculate $T^{5}$ ) Use Equation 6 to calculate $T^{5}$.

Therefore, the relationship between $\Delta T$ and the traffic demand under the SUE-FD condition can be obtained and graphically shown in Figure 3. According to $\Delta T>0$, the range of the traffic demand (RTD) in which the traffic paradox occurs can be deduced as Equation 16 with different values of $\theta$. And it is noteworthy that the flows on path 3 always exist even though the travel cost of path 3 is very high. Due to the network loading property of the SUE, the traffic paradox will persist until the traffic demand reaches the upper bound.

$$
R T D=\left\{\begin{array}{l}
{[3.52,10], \text { if } \theta=0.01} \\
{[3.02,10], \text { if } \theta=0.1} \\
{[2.6,10], \text { if } \theta=1}
\end{array}\right.
$$

As Figure 3 shows, the degree of travelers' perception errors can observably impact the RTD in which the traffic paradox occurs. A counter-intuitive result is that a lower degree of travelers' perception errors may 


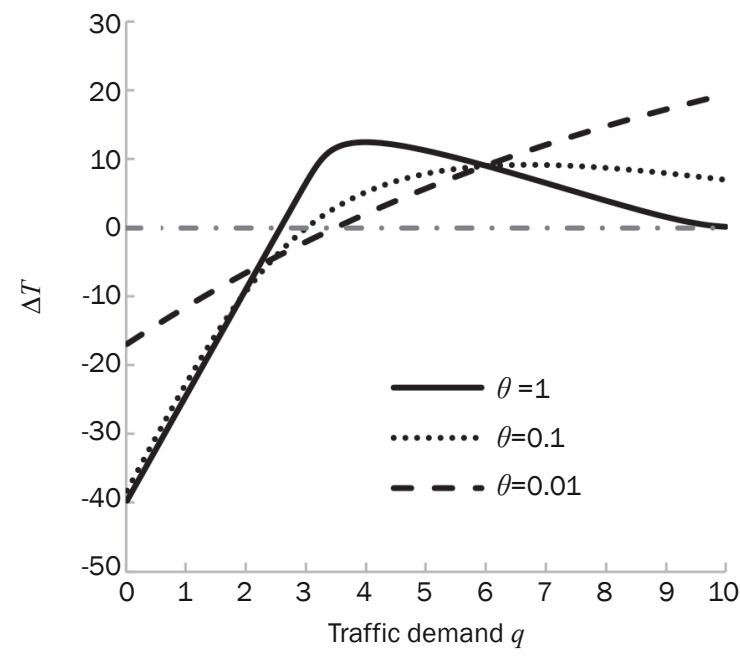

Figure 3 - The values of $\Delta T$ for variable $q$ under SUE-FD

not be a better option. In other words, within a certain range of the traffic demand, providing higher-quality information will lead to a poorer network performance.

To better interpret this result, parameter $\rho$ is set as the proportion of flows on path 3 in the traffic demand. According to $\Delta T \leq 0$, a region of $\rho$ with respect to the traffic demand can be determined by Equation 17, which is the area surrounded by the axes and the critical line as Figure 4 shows. When $\rho$ falls within the indicated area, the traffic paradox will not occur, and the new addition of link 5 can improve (or at least not reduce) the operational efficiency of a network. We can observe that the curve with a lower $\theta$ goes down more gently, but has a lower initial $\rho$, and it intersects the critical line at a higher traffic demand, so the reversed traffic paradox occurs. This analysis is more specific than of the previous studies, with the advantage being that we only need to control $\rho$ within this area through certain management measures (such as route guidance). The operation efficiency of a network will get

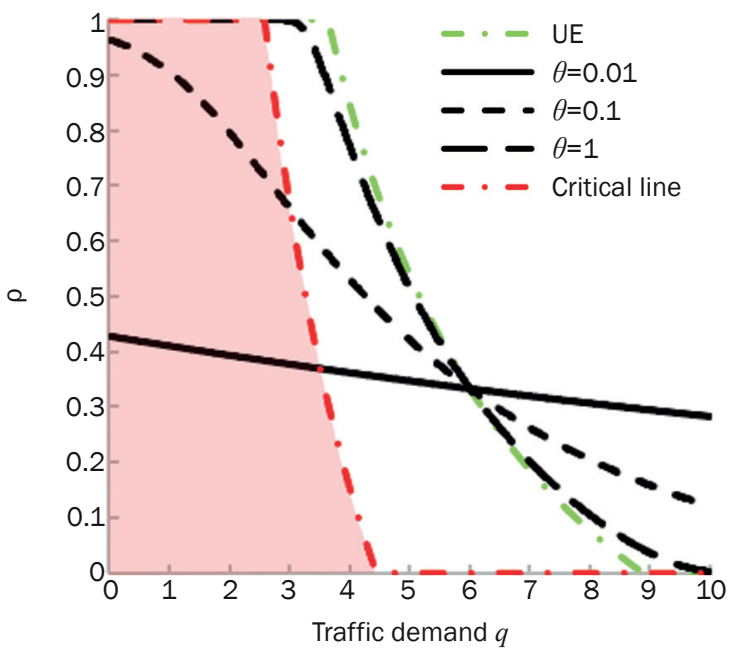

Figure 4 - The values of $\rho$ for variable $q$ with different $\theta$ improved compared to that of the original network without link 5 , even though this is not the system optimization condition.

$\rho \leq \frac{80-18 q}{13 q}$ and $0 \leq \rho \leq 1$

\section{ELASTIC DEMAND}

In reality, traffic demand is not fixed. Instead, it will be influenced by the level of network service. As the travel cost increases, travelers may decide to use a different mode of travel (e.g., subway), shift the time of travel, or even give up this trip. Taking these factors into account, the OD traffic demand can be assumed as a function of the OD travel cost as follows:

$q=D(\mu)$

where $\mu$ represents a certain OD travel cost and it is different under the UE and the SUE. $D(\cdot)$ is the OD traffic demand function, it is expected to be monotonically decreasing (or at least nonincreasing) with regard to the OD travel cost. This function is also bounded from above for the maximum OD traffic demand. In this paper, we use the following linear function:

$q=\bar{q}-\lambda \mu$

where $\bar{q}$ is the upper bound of the OD traffic demand. It is decided by the population size, vehicle ownership for origin nodes, the employment intensity or retail for destination nodes, and the network capacity. $\lambda$ represents the degree of travelers' sensitivity to travel cost, a higher value of $\lambda$ indicates that travelers are more concerned about travel cost, and traffic demand decreases more when travel cost increases, and vice versa. If $\lambda=0$, it implies that the traffic demand is rigid and equivalent to the fixed demand condition.

Under the elastic demand condition, apart from the equilibrium conditions in Section 2, Equation 19 is also needed for a pleasing outcome. Here, another seemingly counter-intuitive and puzzling result is that the addition of links may not induce a higher traffic demand but lead to a lower traffic demand. So, we consider both indexes of $\Delta q\left(\Delta q=q^{5}-q^{4}\right)$ and $\Delta T$ in order to characterize whether the traffic paradox occurs under the elastic demand condition.

\subsection{User equilibrium (UE)}

Under the UE condition, $\mu$ in Equation 19 represents the minimum OD travel cost. By solving the equilibrium conditions 7 and 19, the expression of $q^{4}$ and $T^{4}$ can be derived as Equation 20:

$$
\begin{aligned}
q^{4} & =\frac{2 \bar{q}-100 \lambda}{2+11 \lambda} \\
T^{4} & =\frac{11 \bar{q}+100}{2+11 \lambda}
\end{aligned}
$$



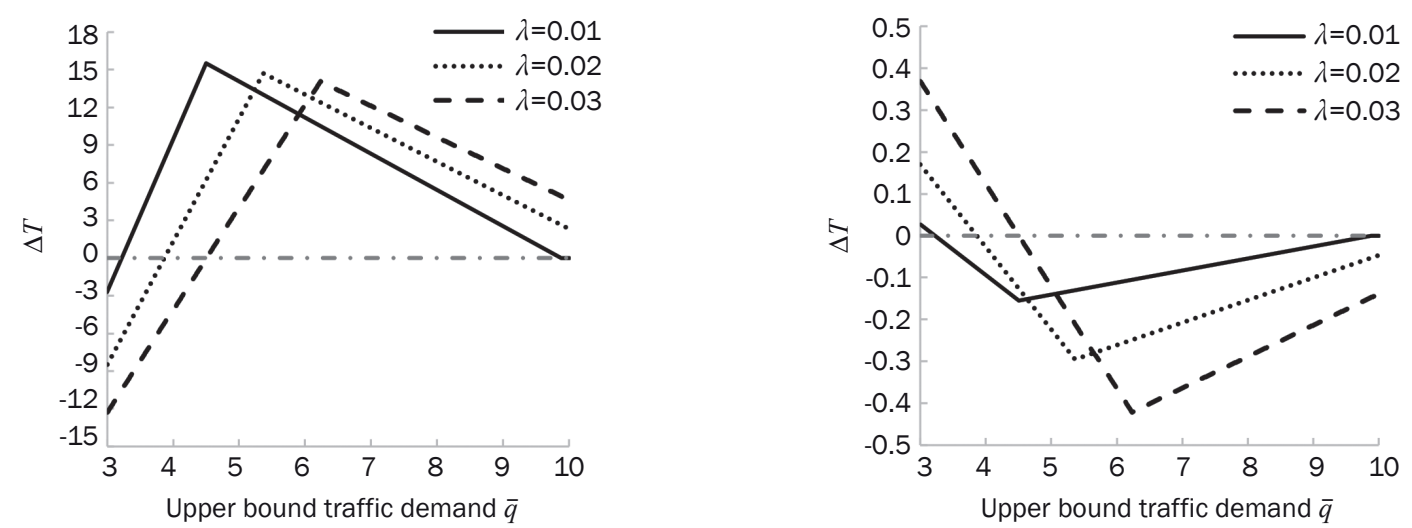

Figure 5 - The values of $\Delta T$ and $\Delta q$ for variable $\bar{q}$ with different $\lambda$

According to different ranges of upper bound of traffic demand (RUBTD), we can also derive the expression of $q^{5}$ and $T^{5}$ using a process similar to the one in Section 3.1, and the results are shown in Equation 21.

$$
\begin{aligned}
q^{5}= & \frac{\bar{q}-10 \lambda}{1+21 \lambda}, T^{5}=\frac{21 \bar{q}+10}{1+21 \lambda}, \text { if } \bar{q} \leq \frac{40+950 \lambda}{11} \\
q^{5}= & \frac{13 \bar{q}-1010 \lambda}{13+31 \lambda}, T^{5}=\frac{31 \bar{q}+1010}{13+31 \lambda}, \\
& \text { if } \frac{40+950 \lambda}{11}<\bar{q}<\frac{80+950 \lambda}{9} \\
q^{5}= & \frac{2 \bar{q}-100 \lambda}{2+11 \lambda}, T^{5}=\frac{11 \bar{q}+100}{2+11 \lambda}, \text { if } \frac{80+950 \lambda}{9}
\end{aligned}
$$

Both $\Delta q$ and $\Delta T$ are observed. However, their results are equivalent to the linear relation between $q^{5}$ and $T^{5}$ (here $T^{5}$ is equal to $\mu_{U E}$ ) in Equation 19. According to Equation 19, the higher the degree of travelers' sensitivity to travel cost, the lower the OD traffic demand. Therefore, the reversed traffic paradox occurs when $\lambda$ increases, as we can observe in Figure 5.

\subsection{Stochastic user equilibrium (SUE)}

As mentioned above, $\mu$ represents different OD travel costs under the UE and the SUE. Under the UE, it is the minimum OD travel cost; under the SUE, it is the expected minimum perceived travel cost, which can be written as [19]:

$$
\mu_{U E}=\frac{-\ln \sum_{k \in K} \exp \left(-\theta c_{k}\right)}{\theta}
$$

In a four-link network, the expressions of $q^{4}$ and $T^{4}$ are:

$$
\begin{aligned}
q^{4} & =\frac{2 \bar{q}+\frac{2 \lambda \ln 2}{\theta}-100 \lambda}{2+11 \lambda} \\
T^{4} & =\frac{100+11 \bar{q}+\frac{11 \lambda \ln 2}{\theta}}{2+11 \lambda}
\end{aligned}
$$

Since it cannot derive the analytical expression of $T^{5}$, a modified method of successive average (MMSA) is developed to solve the SUE-ED model in a five-link network to obtain $q^{5}$ and $T^{5}$.
Step 1. (Initialization) Set tolerance error $\varepsilon$, set the working route $K$, initial traffic flow $f_{k}^{(0)}, x_{a}^{(0)}$ and $q^{(0)}$ based on free flow travel time and initial iteration number $n=1$,

Step 2. (Update travel cost) Use Equations 1-4 to update $c_{k}^{(n)}$ and $t_{a}^{(n)}$, ,

Step 3. (Update traffic flow) Use Equations 14, 15, 19 and 22 to calculate the auxiliary traffic flow $d_{k}^{(n)}$ and traffic demand $l^{(n)}$ to update the traffic flow and demand $f_{k}^{(n+1)}=f_{k}^{(n)}+\frac{1}{n}\left(d_{k}^{(n)}-f_{k}^{(n)}\right), q^{(n+1)}=q^{(n)}+\frac{1}{n}\left(l^{(n)}-q^{(n)}\right)$, Step 4. (Check convergence) If $\frac{\sqrt{\sum_{k \in K}\left(f_{k}^{(n+1)}-f_{k}^{(n)}\right)^{2}}}{\sum_{k \in K} f_{k}^{(n)}}+$ $+\frac{\sqrt{\left(q^{(n+1)}-q^{(n)}\right)^{2}}}{q^{(n)}} \leq \varepsilon$, then stop, $f_{k}^{(n+1)}$ and $q^{(n+1)}$ is the optimal solution, $q^{5}=q^{(n+1)}$. Otherwise, set $\mathrm{n}:=\mathrm{n}+1$, go to step 2,

Step 5. (Calculate $T^{5}$ ) Use Equation 6 to calculate $T^{5}$.

The RUBTDs where the traffic paradox occurs characterized by $\Delta q$ and $\Delta T$ are not equivalent, and their difference may quite strongly depend on the degree of travelers' perception errors $\theta$. Their difference comes from the difference between $\mu_{S U E}$ and the MUTC, as $\mu_{S U E}$ represents the expected minimum perceived travel cost, unlike the MUTC, which makes the relation between $q^{5}$ and $T^{5}$ not linear but more complex. We can observe in Figure 6 that a higher $\theta$ can make the result of a traffic assignment be closer to the UE-ED, which is similar to the conclusion under the FD condition, and the difference between the indexes of $\Delta q$ and $\Delta T$ is smaller. On the contrary, a lower $\theta$ will lead to a more different result, and when $\theta$ is too low, we may get opposite results from the two indexes, which means that it is not the traffic paradox characterized by $\Delta q$ but the traffic paradox characterized by $\Delta T$ which can be seen from the result found in Figure 6 when $\theta=0.01$. When it comes to travelers, travel cost can be more intuitively felt, so $\Delta T$ is a more reasonable index to characterize a traffic paradox than $\Delta q$ under the SUE-ED condition. 

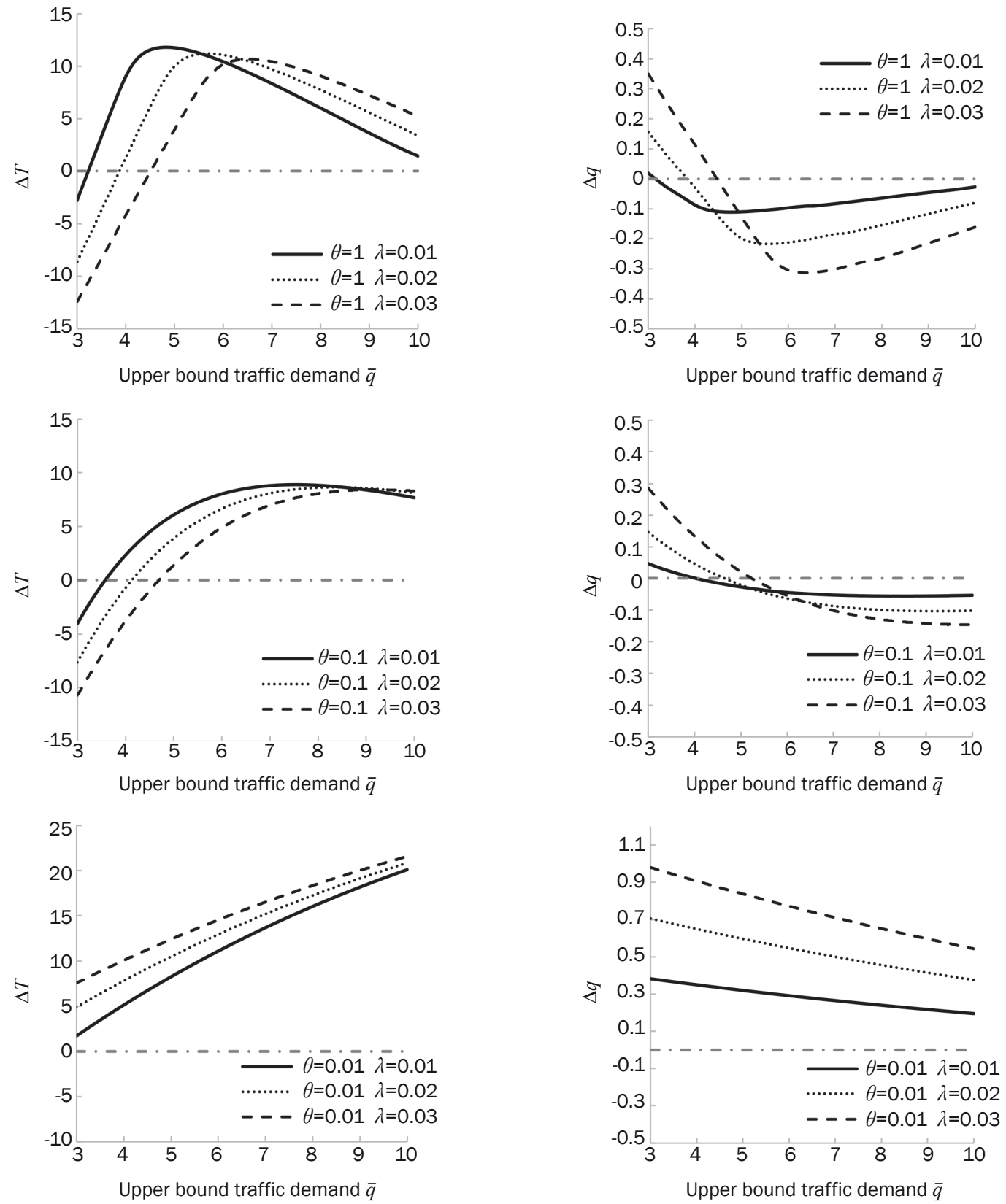

Figure 6 - The values of $\Delta T$ and $\Delta q$ for variable $\bar{q}$ with different $\theta$ and $\lambda$

Besides, we can also observe that each of the curves is not monotonic and the reversed traffic paradox occurs as the parameter of $\lambda$ increases.

\section{CONCLUSIONS}

The traffic paradox on Braess' network under four equilibrium conditions is investigated in this paper. We made some efforts to complete the studies of the traffic paradox under the static traffic assignment conditions, and emphatically analyze the impacts of travelers' perception errors and travel cost sensitivity on the occurrence of a traffic paradox. The applicability of the three indexes and the difference between them, including total travel cost, mean unit travel cost and traffic demand (under the elastic demand) were analyzed in order to characterize whether the traffic paradox occurs. The main conclusions in this paper are as follows:

1) The occurrence of a traffic paradox depends on the traffic demand, as the link travel cost function and equilibrium conditions are given. There is a traffic demand range where the traffic paradox occurs, and if the traffic demand falls outside of the range, the operation efficiency of a new network with addition of links is better than that of the original one.

2) Three indexes, including total travel cost, mean unit travel cost and traffic demand (only under the ED condition) are observed in order to characterize 
whether a traffic paradox occurs. Under the FD condition, the results based on the TTC and the MUTC are equivalent; under the ED condition, the TTC is not applicable, and the results based on the MUTC and the traffic demand are equivalent under the UE condition. However, there is a certain difference which depends on the degree of travelers' perception errors under the SUE condition for its inner property.

3) In a certain range of the traffic demand, higher perception errors may be beneficial to the network performance. In Braess' network, we see that a higher degree of travelers' perception errors can create the RTD in which the reversed traffic paradox occurs, and lower proportion of flows on path 3 in traffic demand can make the network performance better when traffic demand becomes high.

4) If travelers are more sensitive to the travel cost, the traffic demand will be lower under the elastic traffic demand condition, and it can create the reversed traffic paradox under every equilibrium condition.

According to the conclusions, we know that the occurrence of a traffic paradox depends on the traffic demand and flow distribution. Though the network has been built, we can also come up with several traffic management measures to avoid a traffic paradox and improve the operational efficiency of the networks. The simplest method is that, when the traffic demand comes within the range in which the traffic paradox occurs, we close the bad link (i.e., link 5 in this paper), and when the traffic demand falls outside the range, we reopen the closed bad link. However, this may not be the best method. Some other methods, such as route guidance, vehicle restriction on links and link tolls, can also be adopted to guide the travelers' path choice, and make the proportion of flows on bad links fall within the range in which the traffic paradox does not occur. It is necessary to conduct more studies on implementing these measures.

\section{ACKNOWLEDGMENTS}

This research was funded by the National Natural Science Foundation of China (No. 51578150, No. 51378119, No. 51608115, No. 51561135003 and No. 71801115), National key research and development program (No. 2016YFE0206800), Natural Science Foundation of Jiangsu Province (BK20150613), research grants from the Research Grants Council of the Hong Kong Special Administrative Region (Project No. PolyU 15212217), the Hong Kong Scholars Program (Project No. G-YZ1R), and the China Scholarship Council (CSC) Program sponsored by the Ministry of Education in China.

凃强, 博士研究生 ${ }^{1}$

电子邮箱: dqtu_1991@163.com

程琳, 博士 ${ }^{1}$ (通讯作者)
电子邮箱: gist@seu.edu.cn

李大韦, 博士 ${ }^{1,2,3}$

电子邮箱: lidawei@seu.edu.cn

马捷, 博士研究生 ${ }^{1}$

电子邮箱: majie9001@163.com

孙超, 博士 4

电子邮箱: sunchao900217@126.com

1 东南大学交通学院

中国江苏省南京市四牌楼 2 号

2 东南大学城市智能交通江苏省重点实验室

中国江苏省南京市四牌楼 2 号

3 东南大学现代城市交通技术江苏高校协同创新中心 中国江苏省南京市四牌楼2号

4 江苏大学汽车与交通工程学院

中国江苏省镇江市学府大道301号

\section{考虑弹性需求的不同均衡条件下的交通悖论}

\section{摘要}

在交通网络设计和管理中, 交通悖论是一个非常受关 注的现象。现有文献中关于交通悖论的研究主要考虑了固 定需求下的用户均衡和随机用户均衡条件, 且在随机用户 均衡条件中, 路段出行费用通常被设置为常数。然而, 交 通需求是弹性的, 尤其当新路段加入交通网络中，可能会 诱增交通量, 并且路段出行费用会受到路段上交通流量的 影响。本文综合分析了四种不同均衡条件下的交通悖论现 象, 包括固定需求和弹性需求下的用户均衡和随机用户均 衡条件。采用起讫点之间的平均单位出行费用作为判断交 通悖论是否发生的主要指标, 分析了出行者的观测误差和 出行费用敏感度对于交通悖论发生的影响。研究结论表 明：交通悖论的发生取决于交通需求和均衡条件; 更高的 观测误差可能会提升网络的性能; 更高的出行费用敏感度 会使交通悖论的发生延后。最后，本文提出了一些交通管 理策略来避免交通悖论的发生，同时提高网络的性能。

\section{关键字}

交通悖论, 用户均衡, 随机用户均衡, 固定需求, 弹性需 求，相继平均算法

\section{REFERENCES}

[1] Braess D. Über ein Paradoxon aus der Verkehrsplanung. Mathematical Methods of Operations Research. 1968;12(1): 258-268.

[2] Murchland JD. Braess's paradox of traffic flow. Transportation Research. 1970;4(4): 391-394.

[3] Steinberg R, Zangwill WI. The prevalence of Braess' paradox. Transportation Science. 1983;17(3): 301-318.

[4] Dafermos S, Nagurney A. On some traffic equilibrium theory paradoxes. Transportation Research Part B: Methodological. 1984;18(2): 101-110.

[5] Calvert B, Keady G. Braess's paradox and power-law nonlinearities in networks. The ANZIAM Journal. 1993;35(1): 1-22.

[6] Hallefjord A, Jornsten K, Storoy S. Traffic equilibrium paradoxes when travel demand is elastic. Asia-Pacific Journal of Operational Research. 1994;11(1): 41-50.

[7] Ma J, Li D, Cheng L, et al. Link Restriction: Methods of Testing and Avoiding Braess Paradox in Networks Considering Traffic Demands. Journal of Transportation Engineering, Part A: Systems. 2017;144(2): 04017076. 
[8] Pas El, Principio SL. Braess' paradox: Some new insights. Transportation Research Part B: Methodological. 1997;31(3): 265-276.

[9] Yang H, Bell MGH. A capacity paradox in network design and how to avoid it. Transportation Research Part A: Policy and Practice. 1998;32(7): 539-545.

[10] Korilis YA, Lazar AA, Orda A. Avoiding the Braess paradox in non-cooperative networks. Journal of Applied Probability. 1999;36(1): 211-222.

[11] Sheffy Y. Urban transportation networks: equilibrium analysis with mathematical programming methods. Traffic engineering control. Prentice-Hall; 1985.

[12] Zhao C, Fu B, Wang T. Braess paradox and robustness of traffic networks under stochastic user equilibrium. Transportation Research Part E: Logistics and Transportation Review. 2014;61: 135-141.

[13] Arnott R, De Palma A, Lindsey R. Properties of dynamic traffic equilibrium involving bottlenecks, including a paradox and metering. Transportation Science. 1993;27(2): 148-160.
[14] Nagurney A, Qiang Q. A network efficiency measure for congested networks. EPL (Europhysics Letters). 2007;79(3): 38005.

[15] Zhao C. Dynamic Traffic Network Model and Time-Dependent Braess' Paradox. Discrete Dynamics in Nature and Society. 2014;2014: 802129.

[16] Zhao C, Fu B, Wang T. Braess' paradox phenomenon of congested traffic networks. Journal of Transportation Systems Engineering and Information Technology. 2012;4: 023.

[17] Sheffi Y. Urban transportation networks. Englewood Cliffs, NJ: Prentice-Hall; 1985.

[18] Lo HK, Szeto WY. Modeling advanced traveler information services: static versus dynamic paradigms. Transportation Research Part B: Methodological. 2004;38(6): 495-515.

[19] Xu X, Chen A, Zhou Z, et al. A multi-class mean-excess traffic equilibrium model with elastic demand. Journal of Advanced Transportation. 2014;48(3): 203-222. 